\title{
Childhood sexual abuse and the risk for recurrent major depression in Chinese women
}

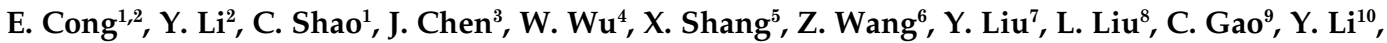 \\ J. Wu ${ }^{11}$, H. Deng ${ }^{12}$, J. Liu ${ }^{13}$, W. Sang ${ }^{14}$, G. Liu ${ }^{15}$, H. Rong ${ }^{16}$, Z. Gan ${ }^{17}$, L. Li ${ }^{18}, K_{.}$Li $^{19}$, J. Pan $^{20}$, Y. Li $^{21}$, \\ Y. Cui ${ }^{22}$, L. Sun ${ }^{23}$, L. Liu ${ }^{24}$, H. Liu ${ }^{25}$, X. Zhao ${ }^{26}$, Y. Zhang ${ }^{27}$, R. Zhang ${ }^{28}$, Y. Chen ${ }^{29}$, X. Wang ${ }^{30}$, H. Li ${ }^{31}$, \\ Y. Chen ${ }^{32}$, Y. Lin ${ }^{33}$, K. S. Kendler ${ }^{34}$, J. Flint ${ }^{2 *}$ and S. Shi ${ }^{1,3}$ \\ ${ }^{1}$ Huashan Hospital, Fudan University, Shanghai, P.R. China ; ${ }^{2}$ Wellcome Trust Centre for Human Genetics, Oxford, UK; ${ }^{3}$ Shanghai Mental \\ Health Centre, Shanghai Jiao Tong University School of Medicine, Shanghai, P.R. China; ${ }^{4}$ Tongji Hospital, Shanghai Tongji University, \\ Shanghai, P.R. China; ${ }^{5}$ Nanjing Brain Hospital, Nanjing, Jiangsu, P.R. China ; ${ }^{6}$ No. 4 Affiliated Hospital of Jiangsu University, Zhenjiang, \\ Jiangsu, P.R. China ; ${ }^{7}$ Tianjin Anding Hospital, Hexi District, Tianjin, P.R. China ; ${ }^{8}$ Shandong Mental Health Centre, Jinan, Shandong, \\ P.R. China; ${ }^{9}$ No. 1 Hospital of Medical College of Xian Jiaotong University, Xi'an, Shaanxi, P.R. China; ${ }^{10}$ No. 1 Hospital of Zhengzhou \\ University, Zhengzhou, Henan, P.R. China; ${ }^{11}$ No. 1 Mental Health Centre Affiliated Harbin Medical University, Nangang District, Harbin, \\ Heilongjiang, P.R. China; ${ }^{12}$ Mental Health Centre of West China Hospital of Sichuan University, Wu Hou District, Chengdu, Sichuan, \\ P.R. China; ${ }^{13}$ Beijing Anding Hospital, Capital Medical University, Xicheng District, Beijing, P.R. China; ${ }^{14}$ Hebei Mental Health Centre, \\ Baoding, Hebei, P.R. China; ${ }^{15}$ Shengjing Hospital of China Medical University, Heping District Shenyang, Liaoning, P.R. China; ${ }^{16}$ Shenzhen \\ Kangning Hospital, Luo Hu, Shenzhen, Guangdong, P.R. China; ${ }^{17}$ No. 3 Affiliated Hospital of Sun Yat-sen University, Tian He District, \\ Guangzhou, Guangdong, P.R. China; ${ }^{18}$ No. 1 Hospital of Shanxi Medical University, Taiyuan, Shanxi, P.R. China; ${ }^{19}$ Mental Hospital of Jiangxi \\ Province, Nanchang, Jiangxi, P.R. China; ${ }^{20}$ The First Affiliated Hospital of Jinan University, Tian He District, Guangzhou, Guangdong, \\ P.R. China; ${ }^{21}$ Wuhan Mental Health Centre, Wuhan, P.R. China ; ${ }^{22}$ No. 3 Hospital of Heilongjiang Province, Beian, Heilongjiang, P.R. China; \\ ${ }^{23}$ Jilin Brain Hospital, Siping, Jilin, P.R. China ; ${ }^{24}$ The First Hospital of China Medical University, He Ping District, Shenyang, Liaoning, P.R. \\ China; ${ }^{25}$ Dalian No. 7 People's Hospital and Dalian Mental Health Centre, Gan Jing Zi District, Dalian, Liaoning, P.R. China; ${ }^{26}$ The First \\ Hospital of Hebei Medical University, Shijiazhuang, Hebei, P.R. China ; ${ }^{27}$ Lanzhou University Second Hospital, Second Clinical Medical College \\ of Lanzhou University, Lanzhou, Gansu Province, P.R. China; ${ }^{28}$ Psychiatric Hospital of Henan Province, Xinxiang, Henan, P.R. China ; \\ ${ }^{29}$ The Fourth Military Medical University affiliated Xijing Hospital, Xi'an, Shaanxi, P.R. China $;{ }^{30}$ No. 4 People's Hospital of Liaocheng, \\ Liaocheng, Shandong, P.R. China; ${ }^{31}$ Guangzhou Brain Hospital/Guangzhou Psychiatric Hospital, Li Wan District, Guangzhou, Guangdong, P.R. \\ China $;{ }^{32}$ Clinical Trial Service Unit, Richard Doll Building, Oxford, UK; ${ }^{33}$ Fuzhou Neuropsychiatric Hospital, Fuzhou City, Fujian, P.R. China ; \\ ${ }^{34}$ Department of Psychiatry, Virginia Commonwealth University, Virginia Institute for Psychiatric and Behavioral Genetics, Richmond, VA, USA
}

Background. Studies in Western countries have repeatedly shown that women with a history of childhood sexual abuse (CSA) are at increased risk for developing major depression (MD). Would this relationship be found in China?

Method. Three levels of CSA (non-genital, genital, and intercourse) were assessed by self-report in two groups of Han Chinese women: 1970 clinically ascertained with recurrent MD and 2597 matched controls. Diagnostic and other risk factor information was assessed at personal interview. Odds ratios (ORs) were calculated by logistic regression and regression coefficients by linear or Poisson regression.

Results. Any form of CSA was significantly associated with recurrent MD [OR 3.26, 95\% confidence interval (CI) 1.95-5.45]. This association strengthened with increasing CSA severity: non-genital (OR 2.47, 95\% CI 1.17-5.23), genital (OR 2.77, 95\% CI 1.32-5.83) and intercourse (OR 13.35, 95\% CI 1.83-97.42). The association between any form of CSA and MD remained significant after accounting for parental history of depression, childhood emotional neglect $(\mathrm{CEN})$, childhood physical abuse (CPA) and parent-child relationship. Among the depressed women, those with CSA had an earlier age of onset, longer depressive episodes and an increased risk for generalized anxiety disorder (GAD; OR 1.92, 95\% CI 1.39-2.66) and dysthymia (OR 2.16, 95\% CI 1.52-3.09).

Conclusions. In Chinese women CSA is strongly associated with MD and this association increases with greater severity of CSA. Depressed women with CSA have an earlier age of onset, longer depressive episodes and increased co-morbidity with GAD and dysthymia. Although reporting biases cannot be ruled out, our results are consistent with the hypothesis that, as in Western countries, CSA substantially increases the risk for MD in China.

Received 2 March 2011; Revised 6 July 2011; Accepted 6 July 2011; First published online 11 August 2011

Key words: Childhood sexual abuse, co-morbidity, major depression.

\footnotetext{
* Address for correspondence: J. Flint, M.D., Wellcome Trust Centre for Human Genetics, Oxford OX3 7BN, UK.

(Email: jf@well.ox.ac.uk) [J. Flint]

(Email shishenxun314@gmail.com) [S. Shi]
}

The online version of this article is published within an Open Access environment subject to the conditions of the Creative Commons Attribution-NonCommercial-ShareAlike licence <http://creativecommons.org/licenses/by-nc-sa/2.5/ > The written permission of Cambridge University Press must be obtained for commercial re-use. 


\section{Introduction}

The causes of major depression (MD) are known only in the broadest outline and it remains a goal of research to define more precisely how this common and debilitating disorder arises. One specific factor known to be strongly associated with risk for MD is childhood sexual abuse (CSA). Numerous studies have demonstrated an association between a history of CSA and MD, with odds ratios (ORs) typically ranging from 2.1 to 7.0 (Jumper, 1995; Neumann et al. 1996; Rind \& Tromovitch, 1997; Fergusson \& Mullen, 1999; Kendler et al. 2000; Paolucci et al. 2001; Kaplow \& Widom, 2007; Young et al. 2007; Bonomi et al. 2008; Carey et al. 2008; Draper et al. 2008; Fergusson et al. 2008; Rohde et al. 2008; Powers et al. 2009; Chen et al. 2010). Furthermore, two studies have applied the co-twin control method to this question and both found significantly increased rates of MD in the exposed versus unexposed twin, thereby substantially increasing the probability that the CSA-MD association is causal (Kendler et al. 2000; Nelson et al. 2002).

However, almost all studies of the impact of CSA on MD have been carried out in Western populations. Doubt remains about the degree to which MD in China differs from that seen in Western countries: the prevalence is lower in China than Western countries, with the 12-month prevalence of MD around $6.6 \%$ in the USA (Kessler et al. 2003) and $2.1 \%$ in China (Phillips et al. 2009). Furthermore, Chinese patients are thought to express emotional distress in a culturally distinct way, preferring to report physical rather than psychological symptoms (Lee et al. 2007). Kleinman (2004) has argued that culture not only influences the experience and diagnosis of MD but also affects the impact of risk factors on MD. If so, CSA might impact differently in Chinese compared to Western populations.

We are aware of four studies that have examined the association between CSA and depressive symptoms in China. Three studies collected information from adolescent girls in school and found a prevalence of CSA in females of $16.7-25.6 \%$. These studies reported higher levels of depressive symptoms in adolescents experiencing CSA that involved physical contact (Chen et al. 2004a, b, 2006). Another study (Sun et al. 2008) found that college students who experienced CSA had higher depression symptom scores as assessed by the Symptom Checklist-90 (SCL-90; Derogatis et al. 1973). However, none of these studies examined the association between CSA exposure and rates of MD.

Understanding how CSA impacts on the risk for MD requires a detailed assessment of CSA, MD and potential confounds. We collected this information in a large case-control study of recurrent MD currently under way in China. Using detailed interviews from patients in 53 hospitals in China, we addressed the following questions. First, what is the magnitude of the association between CSA and MD in Chinese women and does it broadly resemble results from the USA and Europe? Second, to what extent is the association between CSA and MD likely to be causal, versus the results of confounded risk factors? Third, in women with recurrent MD, does the nature and severity of the illness and the pattern of co-morbidity differ between those who have and those who have not reported CSA?

\section{Method}

Sample

The data for the present study were drawn from the ongoing China, Oxford and VCU Experimental Research on Genetic Epidemiology (CONVERGE) study of MD. These analyses were based on a total of 1970 cases recruited from 53 provincial mental health centres and psychiatric departments of general medical hospitals in 41 cities and 19 provinces, and 2597 controls who were recruited from patients undergoing minor surgical procedures at general hospitals or from local community centres.

All cases and controls were female and had four Han Chinese grandparents. Cases and controls were excluded if they had a pre-existing history of bipolar disorder, any type of psychosis or mental retardation. Cases were between 30 and 60 years old, had two or more episodes of MD, with the first episode occurring between ages 14 and 50, and had not abused drugs or alcohol prior to their first depressive episode. Controls were chosen to match the region of origin of cases, were aged between 40 and 60, had never experienced an episode of MD and were not blood relatives of cases. An older minimal age of controls was used to reduce the chances that they might have a subsequent first onset of MD. The mean (S.D.) age of cases and controls in the dataset was similar: $45.1(8.8)$ years for cases and 47.7 (5.5) years for controls.

All subjects were interviewed using a computerized assessment system; an interview lasted on average $2 \mathrm{~h}$ for a case and $1 \mathrm{~h}$ for a control. All interviewers were medical professionals and were trained by the CONVERGE team for a minimum of 1 week in the use of the interview. Seventy-one per cent of the interviewers were fully accredited psychiatrists, $14 \%$ were nurses and $15 \%$ were recently qualified doctors undertaking postgraduate training in psychiatry. The interview includes assessment of demographic factors, psychopathology, psychosocial functioning 
and personal characteristics. Interviews were tape recorded and a proportion of them were listened to by the trained editors who provided feedback on the quality of the interviews. The study protocol was approved centrally by the Ethical Review Board of Oxford University and the ethics committee in participating hospitals in China.

\section{Measures}

A lifetime history of depressive (dysthymia and major depressive disorder) and anxiety disorders [generalized anxiety disorder (GAD) and panic disorder (PD)] was assessed with the Composite International Diagnostic Interview (CIDI; WHO, 1997), which classifies diagnoses according to DSM-IV criteria (APA, 1994). The interview was originally translated into Mandarin by a team of psychiatrists in Shanghai Mental Health Centre, with the translation reviewed and modified by members of the CONVERGE team.

Additional information using instruments developed for the Virginia Adult Twin Study of Psychiatric and Substance Use Disorders (VATSPSUD; Kendler \& Prescott, 2006), translated and reviewed for accuracy by members of the CONVERGE team, was collected on phobia, postnatal depression, stressful life events, CSA, family history and parent-child relationships. Phobia was diagnosed using an adaptation of DSM-III criteria requiring one or more unreasonable fear, and the section is translated from the VATSPSUD study. Information on postnatal depression was assessed using an adaptation of the Edinburgh Scale (Cox et al. 1987). The stressful life events section, also developed for the VATSPSUD study, assessed 16 traumatic lifetime events and the age of their occurrence. The CSA module was a shortened version of the more detailed module used in the VATSPSUD study, which is in turn based on the instrument developed by Martin et al. (1993). We assessed separately the history of MD in mothers and fathers of our cases and controls using the Family History Research Diagnostic criteria (Endicott et al. 1992), and parent-child relationships were measured with the 16-item Parental Bonding Instrument (PBI) modified by Kendler (1996) based on Parker's original 25-item instrument (Parker et al. 1979). Three factors were extracted from these 16 items and labelled warmth, protectiveness and authoritarianism.

Both the case and control interviews were fully computerized into a bilingual system of Mandarin and English developed in Oxford, and called SysQ. Skip patterns were built into SysQ. Interviews were administered by trained interviewers and entered offline in real time onto SysQ, which was installed on laptops.
Once an interview was completed, a backup file containing all the previously entered interview data could be generated with database-compatible format. The backup files together with an audio recording of the interview were uploaded to a designated server currently maintained in Beijing by a service provider. All the uploaded files in the Beijing server were then transferred to an Oxford server.

There is evidence that sensitive subjects such as CSA are more accurately reported with more confidential methods of assessment (Laumann et al. 1994), and therefore participants were asked to fill in a paper questionnaire about CSA (Martin et al. 1993). The questions asked whether, before the subject was 16 years old, did any adult or any other older person involve the subject in any unwanted incidents such as (1) inviting or requesting them to do something sexual, (2) kissing or hugging in a sexual way, (3) touching or fondling private parts, (4) showing their sex organs, (5) making them touch the person in a sexual way, or (6) attempting or having sexual intercourse. The possible responses were 'never', 'once' and 'more than once'. We used these responses to define three forms of CSA (Kendler et al. 2000) : (1) non-genital CSA including sexual invitation, sexual kissing, and exposing (2) genital CSA including fondling and sexual touching and (3) attempted or completed intercourse.

Inter-rater agreement was assessed by repeating the CSA component of the interview for 54 subjects (16 controls and 38 cases, including 14 reporting CSA). We obtained a mean $\kappa$ of 0.73 for three CSA categories (genital: 0.70 ; non-genital: 0.71 ; none: 0.78 ). However, we were able to reinterview only two cases initially reporting intercourse.

We also assessed educational level by dividing the sample into 11 categories, reflecting the highest level of their educational attainment: (1) no education, (2) pre-school education, (3) primary school, (4) junior middle school, (5) senior middle school, (6) technical and vocational school, (7) adult/radio/television schooling, evening education, (8) junior college, (9) bachelor's degree, (10) master's degree and (11) Ph.D.

The results of the fifth Chinese census on 11 November 2000 are presented by the National Bureau of Statistics of China at www.stats.gov.cn/tjsj/ndsj/ renkoupucha/2000pucha/pucha.htm, and tables 1-8 on the education and sex of individuals aged above 6 years can be found at www.stats.gov.cn/tjsj/ndsj/ renkoupucha/2000pucha/html/t0108.htm. In the current study, the distribution of educational attainment was comparable to that of the China census data from 2000. In our sample, $17 \%$ had pre-school or no education $(v .13 \%), 36 \%$ had primary $(v .39 \%), 32 \%$ middle (v. 33\%) and $12 \%$ high school education (v. $10 \%$ ). In addition, in our sample $2 \%$ attended 
Table 1. Childhood sexual abuse (CSA) and the odds ratios for major depression (MD)

\begin{tabular}{lcccc}
\hline & Any CSA & Non-genital CSA & Genital CSA & Intercourse \\
\hline MD $(n=1920)$ & $189(9.8)^{* * *}$ & $67(3.5)^{* * *}$ & $76(4.0)^{* * *}$ & $46(2.4)^{* * *}$ \\
Controls $(n=2588)$ & $70(2.7)$ & $31(1.2)$ & $29(1.1)$ & $10(0.4)$ \\
Model 1 & $3.26(1.95-5.45)^{* * *}$ & $2.47(1.17-5.23)^{*}$ & $2.77(1.32-5.83)^{* *}$ & $13.35(1.83-97.42)^{*}$ \\
Model 2 & $2.63(1.55-4.46)^{* * *}$ & $1.91(0.88-4.16)$ & $2.31(1.07-4.98)^{*}$ & $10.87(1.47-80.38)^{*}$ \\
Model 3 & $1.86(1.06-3.29)^{*}$ & $1.47(0.66-3.29)$ & $1.57(0.66-3.74)$ & $6.99(0.92-53.22)$ \\
\hline
\end{tabular}

Values are given as $n(\%)$ or odds ratio ( $95 \%$ confidence interval).

Model 1 includes variables that reflect age and educational background. Model 2 includes in addition parental family history of depression. Model 3 includes in addition parent-child relationship, childhood physical abuse (CPA) and childhood emotional neglect (CEN).

${ }^{*} p<0.05,{ }^{* *} p<0.01,{ }^{* * *} p<0.001$.

university, compared to $3 \%$ in the census data. However, it should be noted that the depressed women in our study were all ascertained in treatment settings so we cannot claim that our sample is representative of all women with MD in China.

We assessed childhood physical abuse (CPA) through the question in our stressful life events section: 'Were you ever physically abused as a child?' We assessed childhood emotional neglect (CEN) through the question in this section: 'Were you ever seriously neglected as a child?' Emotional neglect refers to a lack of emotional support and inadequate attention to a child's emotional needs, including the need for affection. Physical abuse refers to bodily assaults on a child by an older person that pose a risk of, or result in, injury.

\section{Statistical analysis}

We examined the association between CSA and MD using logistic regression in $\mathrm{R}$ ( $\mathrm{R}$ Development Core Team, 2004), from which we derived estimates of ORs and their associated 95\% confidence intervals (CIs). We examined the degree to which the association between self-reported CSA and MD changed with the inclusion of variables that reflect parental family history of depression, CPA, CEN and parent-child relationships according to the PBI. We controlled only for age and education in the first step of the logistic regression model and then for parental MD history in the second step. In the third step, we added CPA, CEN and PBI. To examine the relationship between CSA and MD co-morbidity, we predicted, in cases only, the risk of depressive patients with CSA having dysthymia, GAD, PD, postnatal depression and phobia with MD, building logistic regression models in three steps.

The association between age at onset and CSA was assessed through linear regression using the age of onset as the dependent variable and any form of CSA as the predictor. We built Poisson regression models using the number of episodes and the duration of the longest episode as the dependent variables, and any form of CSA as predictor. Then we controlled for the covariates step by step as above. Coefficients and $p$ values for these models were obtained using the statistical language R (R Development Core Team, 2004).

\section{Results \\ Prevalence of CSA}

Any form of CSA was reported by $9.8 \%$ of the women with a history of recurrent MD versus $2.7 \%$ of controls. Table 1 shows that the rates of the specific forms of CSA were consistently higher in cases versus controls. In particular, unwanted attempted or completed intercourse before the age of 16 was reported by $2.4 \%$ of women with MD versus $0.4 \%$ of controls.

\section{The relationship between MD and CSA}

Controlling for age and educational background (model 1 in Table 1), any form of CSA was strongly associated with a history of recurrent MD (OR 3.26). Looking at specific forms of CSA, the association with MD strengthened with increasingly severe abuse: non-genital (OR 2.49), genital (OR 2.77) and intercourse (OR 13.35).

We considered whether the relationship between CSA and MD reflected the presence of mental illness in the parents. On average, parents with psychiatric illness provide poorer environments for their children, potentially not attending to their daughters' wellbeing and so increasing the risk of abuse. Controlling for a parental diagnosis of MD in the CSA analyses eliminates that possible confounding effect. We therefore repeated the analyses, adding in family history diagnoses of mother and father (model 2 in Table 1). 
Table 2. Childhood sexual abuse (CSA) and the odds ratios for co-morbid disorders

\begin{tabular}{lcclll}
\hline & Any CSA & No CSA & Model A & Model B & Model C \\
\hline MD and dysthymia $(n=349)$ & $59(17.4)$ & $280(82.6)$ & $2.16^{* * *}(1.52-3.09)$ & $2.04^{* * *}(1.42-2.92)$ & $1.98(1.30-3.02)^{* *}$ \\
MD and postnatal depression $(n=359)$ & $58(16.2)$ & $301(83.8)$ & $1.69^{* *}(1.16-2.46)$ & $1.61^{*}(1.10-2.34)$ & $1.53(0.99-2.35)$ \\
MD and GAD $(n=568)$ & $84(14.8)$ & $484(85.2)$ & $1.92^{* * *}(1.39-2.66)$ & $1.87^{* * *}(1.35-2.59)$ & $1.61^{*}(1.11-2.35)$ \\
MD and PD $(n=190)$ & $27(14.2)$ & $163(85.8)$ & $1.72^{*}(1.08-2.74)$ & $1.67^{*}(1.05-2.68)$ & $1.45(0.83-2.52)$ \\
MD and phobia $(n=1427)$ & $151(10.6)$ & $1276(89.4)$ & $2.04^{* * *}(1.46-2.84)$ & $1.76^{* *}(1.25-2.48)$ & $1.32(0.90-1.94)$ \\
\hline
\end{tabular}

GAD, Generalized anxiety disorder; PD, panic disorder.

Values given as $n(\%)$ or odds ratio ( $95 \%$ confidence interval).

Model A includes variables that reflect age and educational background. Model B includes in addition parental family history of depression. Model C includes in addition parent-child relationship, childhood physical abuse (CPA) and childhood emotional neglect CEN).

${ }^{*} p<0.05,{ }^{* *} p<0.01,{ }^{* * *} p<0.001$.

The magnitude of the association between CSA and MD decreased, but remained significant.

CSA is also correlated with childhood adversity, in particular CPA and CEN, and with the nature of the relationship between parent and child. To explore whether parent-child relationships and childhood adversity explain the association between CSA and MD, we added into model 3 (from Table 1) the results from the PBI, and the assessments of CPA and CEN. We found that the OR for the effect of CSA on MD was mildly decreased (Table 1). That is, controlling for the parent-child relationship, CPA and CEN, any form of CSA remained significantly associated with a history of recurrent MD in our sample.

\section{The relationship between the CSA and co-morbidity and phenomenology of MD}

We next considered the possibility that the experience of CSA could increase the rate of co-morbid disorders in patients with MD. We examined the association between self-reported CSA and MD with dysthymia, postnatal depression, GAD, PD and phobia. Within cases, controlling only for age and education (Table 2, model A), a lifetime history of GAD, dysthymia, PD, postnatal depression and phobia was associated with CSA $(p<0.05)$. These associations all remained significant with the inclusion of parental MD history (Table 2, model B). Adding PBI, CPA and CEN into the model, the association between CSA and dysthymia and between CSA and GAD remained significant $(p<0.05)$.

The experience of CSA could alter the form and frequency of $\mathrm{MD}$. We examined this question by looking at these features of MD in our cases. Within cases, controlling only for age and education (Table 3, model A), we found that depressed patients with CSA had a significantly earlier age of onset of MD. This result was significant $(p=0.007)$ when we included parental history of MD (model B) and when including parent-child relationship, CPA and CPN in model C $(p=0.03)$. The duration of the longest episode of MD for those with CSA was significantly greater than those without CSA $(p<0.001)$ and this was seen in all models. However, no relationship was found between CSA and the number of reported depressive episodes in models A and B. After controlling for parent-child relationship, CPA and CEN, the result became significant $(p=0.018)$ (Table 3$)$.

\section{Discussion}

Our study of the relationship between CSA and MD in Han Chinese women has produced four major findings. First, in this population, CSA is robustly associated with an increased risk of developing MD. Furthermore, CSA was found to have a systematic 'dose-response' relationship with risk for MD: the greater the severity of CSA, the stronger is the observed association with MD. Second, controlling for potential confounders, specifically parental history of MD, parent-child relationship and other childhood adversities attenuated the CSA-MD association but the association between $\mathrm{MD}$ and 'any CSA' still remained significant. These findings increase the probability that the association between CSA and MD in our sample is a causal one. Third, in depressed patients, CSA impacted on patterns of co-morbidity. In women with recurrent $\mathrm{MD}$, those with a history of CSA were significantly more likely to also suffer from dysthymia, post-natal depression, GAD, phobia and probably PD. Fourth, in depressed women, CSA is modestly associated with an earlier age of onset of MD and strongly associated with longer depressive episodes.

The association of CSA with risk of MD has been extensively documented in Western populations 
Table 3. The relationship between childhood sexual abuse (CSA) and clinical features of major depression (MD)

\begin{tabular}{|c|c|c|c|c|c|c|c|c|c|}
\hline & \multicolumn{3}{|c|}{ Age of onset of $\mathrm{MD}^{\mathrm{a}}$} & \multicolumn{3}{|c|}{ No. of episodes of $\mathrm{MD}^{\mathrm{b}}$} & \multicolumn{3}{|c|}{ Duration of the longest episode of $\mathrm{MD}^{\mathrm{c}}$} \\
\hline & $\mathrm{A}$ & B & C & A & B & C & A & B & C \\
\hline $\begin{array}{l}\text { Coefficient }{ }^{\mathrm{d}} \\
p \text { value }\end{array}$ & $\begin{array}{r}-1.70 \\
0.006\end{array}$ & $\begin{array}{r}-1.68 \\
0.007\end{array}$ & $\begin{array}{r}-1.51 \\
0.03\end{array}$ & $\begin{array}{l}0.07 \\
0.107\end{array}$ & $\begin{array}{l}0.04 \\
0.293\end{array}$ & $\begin{array}{l}0.11 \\
0.018\end{array}$ & $\begin{array}{l}0.06 \\
3.59 \times 10^{-6}\end{array}$ & $\begin{array}{l}0.06 \\
3.20 \times 10^{-6}\end{array}$ & $\begin{array}{l}0.07 \\
1.38 \times 10^{-6}\end{array}$ \\
\hline
\end{tabular}

Model A includes variables that reflect age and educational background. Model B includes in addition parental family history of depression. Model C includes in addition parent-child relationship, childhood physical abuse (CPA) and childhood emotional neglect CEN).

a The results from a linear regression model with age of onset of MD as response and with 'any CSA' as predictor.

b The results from a Poisson regression model with numbers of episodes of MD as response and with 'any CSA' as predictor.

' The results from a Poisson regression model with duration of the longest episodes of MD as response and 'any CSA' as predictor.

${ }^{d}$ The predicted change in the age of onset (in years) of MD for cases who suffered CSA.

(Fergusson et al. 1996; Mullen et al. 1996; Fergusson \& Mullen, 1999; Fleming et al. 1999; Kendler et al. 2000; Nelson et al. 2002), as has the general dose-response relationship between CSA and risk for developing MD (Fergusson et al. 1996; Kendler et al. 2000), and also the finding that CSA involving intercourse is the most strongly associated with risk for MD (Fergusson et al. 1996; Kendler et al. 2000 ; Nelson et al. 2002; Jonas et al. 2010). Our findings indicate that these observations about the relationship between MD and CSA also hold true in China.

CSA could be an indirect or a direct manifestation of parental depressive disorder. Based on our analyses, neither is a likely explanation because the association between CSA and MD in children still remained significant when we controlled for parental MD history. This result is again consistent with Western studies (Kendler et al. 2000; Nelson et al. 2002). Some researchers have reported that control and alienation in the relationship between parent and child increases the risk of developing MD (Denollet et al. 2007). Others have reported that CPA and CEN in part explain the effect of CSA on MD (Cohen et al. 2001; Fergusson et al. 2008; Spinhoven et al. 2010). In our study, when we controlled for the effect of parent-child relationship, CPA and CEN, we found that the strength of the relationship between the risk of $\mathrm{MD}$ and any CSA remained significant. Our analysis indicates that CSA increases the risk of depression in part independently of the effects of the parent-child relationship, physical abuse and emotional neglect.

Support for the causal role of CSA on MD comes from the observation that increasing severity of abuse is associated with an increased risk of MD. However it should be noted that the increase in OR between non-genital (2.47) and genital (2.77) CSA is relatively modest, whereas there is a large increase for intercourse (13.35). This indicates that there may be a non-linear relationship between the degree of abuse and outcome. In this context, it should also be noted that exposure to multiple forms of abuse, including physical and emotional neglect, also increases the risk of MD. Thus, although the evidence seems to be consistent with a causal model, we cannot exclude more complex relationships between CSA and MD.

We also considered whether the effect of CSA on MD might result in characteristic symptomatology. The greater prevalence of co-morbid GAD, dysthymia, PD, post-natal depression and phobia, together with our observation of an earlier age of MD onset and longer periods of $\mathrm{MD}$, indicates that this may be true. One study has shown that patients suffering from anxiety disorders co-morbid with MD had increased rates of childhood abuse, including CSA (Safren et al. 2002). Two previous studies have also studied the relationship between childhood adversities and an earlier age of onset of MD (Young et al. 1997; Bernet \& Stein, 1999). In one study (using 47 adults with MD and 41 comparison subjects), CEN predicted an earlier age of onset of MD. In the other, CSA was associated with an age of onset about 11 years earlier using 650 clinical cases (Young et al. 1997). Both studies failed to control for confounders in exploring the relationship.

Our findings replicate those found in other populations and indicate that, at least in some respects, the aetiology and phenomenology of MD in China is similar to that reported elsewhere. One interpretation of our results is that MD in China is more similar to MD in Western populations than has been reported previously (Kleinman, 2004).

Nevertheless, there is evidence that MD in China differs from MD in Western populations. Epidemiological research has shown that the prevalence of MD in China is lower than that reported in Western countries (Andrade et al. 2003; Kessler et al. 2003; Lee et al. 2009; Phillips et al. 2009). One study reported that 
the lifetime prevalence of MD was 3.6\% (Lee et al. 2009), far less than the prevalence in Western countries, where the lifetime prevalence of MD was reported to be around 17\% (Kessler et al. 1994, 2003). Furthermore, there is evidence that the symptomatology is different. A clinical study in China showed that depressed patients prefer to report physical symptoms such as heart-related symptoms, pain, and sleeplessness (Lee et al. 2007). Chinese patients tend to deny depression or express it somatically (Parker et al. 2001), believing depression to be a psychiatric disease, which is stigmatized in Chinese culture. Even Chinese patients in Western countries have less recourse to mental health services when they have a mental disease than Western patients (Chen et al. 2009).

Our study has several potential limitations. First, as in most epidemiological studies, CSA in our sample was assessed retrospectively. Recall may be inaccurate and/or biased. Estimates for the rates of CSA in our control sample $(2.7 \%$ for any CSA and only $0.4 \%$ for unwanted intercourse) are lower than estimates obtained from other, non-Chinese samples [24.7-30.4\% for any CSA and 5.6-8.4\% for intercourse (Kendler et al. 2000; Nelson et al. 2002; Dube et al. 2005)]. These low rates may reflect either under-reporting in Chinese populations or truly lower rates of CSA in China versus most Western populations.

The one previous study in China reporting rates of CSA found figures very similar to those in our sample. Chen et al. (2004a) found CSA involving intercourse to be reported by $0.3 \%$ in 1155 female students. Subjects reported CSA in a self-report questionnaire, so the low rates of CSA in our sample were not the result of hesitance to admit to CSA during a face-to-face interview. If cultural factors influenced subjects to underreport CSA, the general expectation is that this bias would impact equally on our cases and controls. However, we cannot rule out the possibility that our cases (because of depressed mood or greater contact with health professionals) were more willing to report CSA than were our controls. If this were true, our observed association between CSA and MD could arise from biased reporting rather than a true causal association.

Second, because of the retrospective nature of our data, we cannot determine whether the association between reporting CSA and a history for MD is the result of a passive gene-environment correlation in which, for example, parents with a genetic risk for MD provide poorer supervision for their children, thereby increasing their risk for CSA, and pass on to them their risk genes for MD.

Third, our assessments of CEN and CPA used in each case a single item. Despite covering important potential traumas, our coverage was far from exhaustive. It is possible that our aspects of the home environment predisposed to both CSA and MD, thereby biasing upwards our estimates of their association.

Fourth, our assessment of socio-economic status was based on the highest educational attainment of study participants. We did not assess additional family characteristics, such as personality and cognitive abilities, which might confound the relationship between CSA and MD.

Fifth, our sample had recurrent MD and consisted of women identified in treatment centres. The impact of CSA on individuals with a single episode of MD who do not present for treatment may be different; we do not know whether our results generalize to other groups of MD patients.

In summary, we have found that CSA, although more rarely reported in Chinese than in most Western samples, is strongly associated with recurrent MD in Chinese women. The association shows a strong doseresponse relationship and is mildly attenuated when controls are added for parental depression and other childhood environmental adversities. These results suggest, but do not prove, that the CSA-MD association in China is causal, as has been suggested in US and Australian samples (Kendler et al. 2000; Nelson et al. 2002). Consistent with other results emerging from the CONVERGE project and other investigations of MD in China, the results of the present paper contribute to a growing literature suggesting far more similarities than differences in the aetiological processes leading to depressive illness in China and in Western populations.

\section{Acknowledgements}

This work was funded by the Wellcome Trust. The authors are part of the CONVERGE consortium (China, Oxford and VCU Experimental Research on Genetic Epidemiology) and gratefully acknowledge the support of all partners in hospitals across China.

\section{Declaration of Interest}

None.

\section{References}

Andrade L, Caraveo-Anduaga JJ, Berglund P, Bijl RV, De Graaf R, Vollebergh W, Dragomirecka E, Kohn R, Keller M, Kessler RC, Kawakami N, Kilic C, Offord D, Ustun TB, Wittchen HU (2003). The epidemiology of major depressive episodes: results from the International Consortium of Psychiatric Epidemiology (ICPE) Surveys. 
International Journal of Methods in Psychiatric Research 12, 3-21.

APA (1994). Diagnostic and Statistical Manual of Mental Disorders. American Psychiatric Association: Washington, DC

Bernet CZ, Stein MB (1999). Relationship of childhood maltreatment to the onset and course of major depression in adulthood. Depression and Anxiety 9, 169-174.

Bonomi AE, Cannon EA, Anderson ML, Rivara FP, Thompson RS (2008). Association between self-reported health and physical and/or sexual abuse experienced before age 18. Child Abuse Journal of Neglect 32, 693-701.

Carey PD, Walker JL, Rossouw W, Seedat S, Stein DJ (2008). Risk indicators and psychopathology in traumatised children and adolescents with a history of sexual abuse. European Journal of Child and Adolescent Psychiatry 17, 93-98.

Chen AW, Kazanjian A, Wong H (2009). Why do Chinese Canadians not consult mental health services: health status, language or culture? Transcultural Psychiatry 46, 623-641.

Chen J, Dunne MP, Han P (2004a). Child sexual abuse in China: a study of adolescents in four provinces. Child Abuse and Neglect 28, 1171-1186.

Chen J, Dunne MP, Han P (2006). Child sexual abuse in Henan province, China: associations with sadness, suicidality, and risk behaviors among adolescent girls. Journal of Adolescent Health 38, 544-549.

Chen JQ, Han P, Dunne MP (2004b). Child sexual abuse: a study among 892 female students of a medical school [in Chinese]. Zhonghua Er Ke Za Zhi 42, 39-43.

Chen LP, Murad MH, Paras ML, Colbenson KM, Sattler AL, Goranson EN, Elamin MB, Seime RJ, Shinozaki G, Prokop LJ, Zirakzadeh A (2010). Sexual abuse and lifetime diagnosis of psychiatric disorders: systematic review and meta-analysis. Mayo Clinic Proceedings 85, 618-629.

Cohen P, Brown J, Smaile E (2001). Child abuse and neglect and the development of mental disorders in the general population. Development and Psychopathology 13, 981-999.

Cox JL, Holden JM, Sagovsky R (1987). Detection of postnatal depression. Development of the 10-item Edinburgh Postnatal Depression Scale. British Journal of Psychiatry 150, 782-786.

Denollet J, Smolderen KG, van den Broek KC, Pedersen SS (2007). The 10-item Remembered Relationship with Parents (RRP10) scale: two-factor model and association with adult depressive symptoms. Journal of Affective Disorders 100, 179-189.

Derogatis LR, Lipman RS, Covi L (1973). SCL-90: an outpatient psychiatric rating scale - preliminary report. Psychopharmacology Bulletin 9, 13-28.

Draper B, Pfaff JJ, Pirkis J, Snowdon J, Lautenschlager NT, Wilson I, Almeida OP (2008). Long-term effects of childhood abuse on the quality of life and health of older people: results from the Depression and Early Prevention of Suicide in General Practice Project. Journal of the American Geriatrics Society 56, 262-271.

Dube SR, Anda RF, Whitfield CL, Brown DW, Felitti VJ, Dong M, Giles WH (2005). Long-term consequences of childhood sexual abuse by gender of victim. American Journal of Preventive Medicine 28, 430-438.
Endicott J, Andreasen N, Spitzer RL (1992). Family History-Research Diagnostic Criteria. Biometrics Research, New York State Psychiatric Institute: New York.

Fergusson DM, Boden JM, Horwood LJ (2008). Exposure to childhood sexual and physical abuse and adjustment in early adulthood. Child Abuse and Neglect 32, 607-619.

Fergusson DM, Horwood LJ, Lynskey MT (1996). Childhood sexual abuse and psychiatric disorder in young adulthood: II. Psychiatric outcomes of childhood sexual abuse. Journal of the American Academey of Child and Adolescent Psychiatry 35, 1365-1374.

Fergusson DM, Mullen PE (1999). Childhood Sexual Abuse: An Evidence-Based Perspective. Sage Publications: Thousand Oaks, CA.

Fleming J, Mullen PE, Sibthorpe B, Bammer G (1999). The long-term impact of childhood sexual abuse in Australian women. Child Abuse and Neglect 23, 145-159.

Jonas S, Bebbington P, McManus S, Meltzer H, Jenkins R, Kuipers E, Cooper C, King M, Brugha T (2010). Sexual abuse and psychiatric disorder in England: results from the 2007 Adult Psychiatric Morbidity Survey. Psychological Medicine 41, 1-11.

Jumper SA (1995). A meta-analysis of the relationship of child sexual abuse to adult psychological adjustment. Child Abuse and Neglect 19, 715-728.

Kaplow JB, Widom CS (2007). Age of onset of child maltreatment predicts long-term mental health outcomes. Journal of Abnormal Psychology 116, 176-187.

Kendler KS (1996). Parenting: a genetic-epidemiologic perspective. American Journal of Psychiatry 153, 11-20.

Kendler KS, Bulik CM, Silberg J, Hettema JM, Myers J, Prescott CA (2000). Childhood sexual abuse and adult psychiatric and substance use disorders in women: an epidemiological and cotwin control analysis. Archives of General Psychiatry 57, 953-959.

Kendler KS, Prescott CA (2006). Environment, and Psychopathology: Understanding the Causes of Psychiatric and Substance Use Disorders. Guildford Press: New York.

Kessler RC, Berglund P, Demler O, Jin R, Koretz D, Merikangas KR, Rush AJ, Walters EE, Wang PS (2003). The epidemiology of major depressive disorder: results from the National Comorbidity Survey Replication (NCS-R). Journal of the American Medical Association 289, 3095-3105.

Kessler RC, McGonagle KA, Zhao S, Nelson CB, Hughes M, Eshleman S, Wittchen HU, Kendler KS (1994). Lifetime and 12-month prevalence of DSM-III-R psychiatric disorders in the United States. Results from the National Comorbidity Survey. Archives of General Psychiatry 51, 8-19.

Kleinman A (2004). Culture and depression. New England Journal of Medicine 351, 951-953.

Laumann EO, Gagnon JH, Michael RT, Michaels S (1994). The Social Organization of Sexuality. University of Chicago Press: Chicago, IL.

Lee DT, Kleinman J, Kleinman A (2007). Rethinking depression: an ethnographic study of the experiences of depression among Chinese. Harvard Review of Psychiatry 15, 1-8. 
Lee S, Tsang A, Huang YQ, He YL, Liu ZR, Zhang MY, Shen YC, Kessler RC (2009). The epidemiology of depression in metropolitan China. Psychological Medicine 39, 735-747.

Martin J, Anderson J, Romans S, Mullen P, O'Shea M (1993). Asking about child sexual abuse: methodological implications of a two stage survey. Child Abuse and Neglect 17, 383-392.

Mullen PE, Martin JL, Anderson JC, Romans SE, Herbison GP (1996). The long-term impact of the physical, emotional, and sexual abuse of children: a community study. Child Abuse and Neglect 20, 7-21.

Nelson EC, Heath AC, Madden PA, Cooper ML, Dinwiddie SH, Bucholz KK, Glowinski A, McLaughlin T, Dunne MP, Statham DJ, Martin NG (2002). Association between self-reported childhood sexual abuse and adverse psychosocial outcomes: results from a twin study. Archives of General Psychiatry 59, 139-145.

Neumann DA, Houskamp BM, Pollock VE, Briere J (1996). The long-term sequelae of childhood sexual abuse in women: a meta-analytic review. Child Maltreatment 1, 6-16.

Paolucci EO, Genuis ML, Violato C (2001). A meta-analysis of the published research on the effects of child sexual abuse. Journal of Psychology 135, 17-36.

Parker G, Gladstone G, Chee KT (2001). Depression in the planet's largest ethnic group: the Chinese. American Journal of Psychiatry 158, 857-864.

Parker G, Tupling H, Brown LB (1979). A Parental Bonding Instrument. British Journal of Medical Psychology 52, 10.

Phillips MR, Zhang J, Shi Q, Song Z, Ding Z, Pang S, Li X, Zhang Y, Wang Z (2009). Prevalence, treatment, and associated disability of mental disorders in four provinces in China during 2001-05: an epidemiological survey. Lancet 373, 2041-2053.
Powers A, Ressler KJ, Bradley RG (2009). The protective role of friendship on the effects of childhood abuse and depression. Depression and Anxiety 26, 46-53.

R Development Core Team (2004). A Language and Environment for Statistical Computing. R Foundation for Statistical Computing: Vienna.

Rind B, Tromovitch P (1997). A meta-analytic review of findings from national samples on psychological correlates of child sexual abuse. Journal of Sex Research 34, 237-255.

Rohde P, Ichikawa L, Simon GE, Ludman EJ, Linde JA, Jeffery RW, Operskalski BH (2008). Associations of child sexual and physical abuse with obesity and depression in middle-aged women. Child Abuse and Neglect 32, 878-887.

Safren SA, Gershuny BS, Marzol P, Otto MW, Pollack MH (2002). History of childhood abuse in panic disorder, social phobia, and generalized anxiety disorder. Journal of Nervous and Mental Disease 190, 453-456.

Spinhoven P, Elzinga BM, Hovens JG, Roelofs K, Zitman FG, van Oppen P, Penninx BW (2010). The specificity of childhood adversities and negative life events across the life span to anxiety and depressive disorders. Journal of Affective Disorders 126, 103-112.

Sun YP, Zhang B, Dong ZJ, Yi MJ, Sun DF, Shi SS (2008). Psychiatric state of college students with a history of childhood sexual abuse. World Journal of Pediatrics 4 , 289-294.

WHO (1997). Composite International Diagnostic Interview (CIDI), Version 2.1. World Health Organization: Geneva. Young EA, Abelson JL, Curtis GC, Nesse RM (1997). Childhood adversity and vulnerability to mood and anxiety disorders. Depression and Anxiety 5, 66-72.

Young MS, Harford KL, Kinder B, Savell JK (2007). The relationship between childhood sexual abuse and adult mental health among undergraduates: victim gender doesn't matter. Journal of Interpersonal Violence 22, 1315-1331. 